\title{
Über die Wirkung des Pilokarpins auf den Darm. Ist das Pilokarpin ausschliesslich ein Parasympathikusgift oder zugleich auch ein Sympathikusgift?
}

\author{
Von \\ Masao Fujlta. \\ Aus dem pharmakologischen Institut, Okayama, Japan \\ (Vorstand: Prof. Dr. K. Okushima).
}

In der modernen Pharmakologie kennt man das Pilokarpin als den Vertreter der parasympathikuserregenden Gifte. Es fördert also, wie von vielen Autoren sowohl am lebenden Tiere als auch am ausgeschnittenen Organe nachgewiesen worden ist, die Bewegungen des Darmes und des Uterus, ja es kann sogar krampfartige Kontraktionen verursachen. Auch fast alle anderen glattmuskeligen Organe des Warmblüters werden durch Pilokarpin stark erregt. Doch sind zuweilen auch andere Wirkungen beobachtet worden. So hat z. B. Gohara' durch seine Versuche gefunden, dass das Pilokarpin auf die Kaninchentube keine erregende Wirkung hervorruft. Und Takaori' ${ }^{2}$ hat an der ausgeschnittenen Milz beobachtet, dass das Pilokarpin auf den Tonus einen hemmenden Einfluss ausübt.

Dagegen ist die Wirkung dieses Giftes am Kaltblüter eine andere. Harnack und Meyer ${ }^{3)}$ haben am Froschherzen nachgewiesen, dass das Pilokarpin auf die zwischen dem Vagusstamm und den Endapparaten liegende Ganglienstation zuerst fördernd, dann aber lähmend wirkt. Nach

1) Gohara, A.; Kinki Fujinka-Gakkai Kaiho, Nr. 7, S. 368.

2) Takaori, S. ; Kyoto Igaku Zasshi, Bd. 20, S. 329.

3) Harnack, E. u. Meyer, H. ; Arch. f. exp. Path. u. Pharm., Bd. 12, S. 366, 1880. 
den Versuchen von Fujitani ${ }^{1)}$ und Kondo ${ }^{2)}$ werden Magen und Ösophagus eines Kaltblüters (Frosch) durch 0,1 \% jges Pilokarpin zuerst erregt und dann sofort gelähmt. Kondo beobachtete auch, dass der Froschdarm schon durch mittlere Pilokarpindosen gehemmt wird.

Am Warmblüterdarm hat man jedoch noch niemals eine hemmende Wirkung des Pilokarpins gefunden und deshalb bis heute allgemein angenommenen, dass das Pilokarpin auf den Darm infolge der Erregung der Parasympathikusendigungen nur erregend wirke. Aus der hier geschilderten hemmenden Wirkung des Pilokarpins auf verschiedene glattmuskelige Organe schien es mir jedoch keineswegs unwahrscheinlich, dass das Pilokarpin neben der parasympathikuserregenden Wirkung auch auf den hemmenden Apparat im Darme erregend wirke, obwohl diese hemmede Wirkung von jener stark erregenden Wirkung verdeckt sein könnte. Um diese Frage zu beantworten, ist es nötig, die Pilokarpinwirkung am atropinisierten Darm genau zu untersuchen, und zwar nach vollstāndiger Lähmung der Parasympathikusendigungen, die nach heutiger Ansicht der einzige Angriffspunkt des Pilokarpins sind.

Magnus $^{3)}$ hat bereits am Katzendünndarm genau beobachtet, wie das Pilokarpin auf mit Atropin vorbehandelte Präparate wirkt. Nach 20 Minuten langer Einwirkung von 0,05 g Atropin (in $200 \mathrm{ccm}$ Ringerlösung) wurde durch das Pilokarpin noch eine maximale und nach 1/2 Stunde immer noch eine zwar schwāchere, aber deutliche Tonussteigerung hervorgerufen. Nach $1 \frac{1}{2}$ stündiger Einwirkung der gleichen Atropindose aber waren die Bewegungen schon recht gering, und 0,025 g Pilokarpin waren dann nicht mehr imstande, eine Tonussteigerung hervorzurufen. Kress ${ }^{4)}$ dagegen fand am Kaninchendünndarm nach vorheriger schwacher Atropinvergiftung (0,0125 $-0,05 \mathrm{~g}$ in $200 \mathrm{ccm}$ Ringerlösung) keine Pilokarpinerregung. Neuere Untersuchungen pflegen die Resultate von Kress zu bestätigen. Ich habe auch in meinen Versuchen am Hühnerdünndarm konstatiert, dass das Pilokarpin an den schwach atropinisierten Darmstücken $(0,001-0,01 \mathrm{~g}$ in 100

1) Fujitani, Y.; Kyoto Igaku Zasshi, Bd. 6, S. 195.

2) Kondo, S.; Kyoto Igaku Zasshi, Bd. 16, S. 663.

3) Magnus, R. ; Pfliger's Arch. f. d. ges. Physiol., Bd. 108, S. 1, 1905.

4) Kress, K.; Ebenda., Bd. 109, S. 603, 1905. 
ccm Ringerlösung) keine erregende Wirkung mehr zeigt. ${ }^{1)}$

Nach diesen Ergebnissen zeigt also das Pilokarpin am atropinisierten Darme eine mehr oder weniger starke Tonuszunahme oder gar keine Wirkung. Die Wirkung des Pilokarpins wird hier also nur bestätigt. Sie erklärt sich aus der Erregung des Parasympathikus im Darm. Da mir aber bei Versuchen am ausgeschnittenen Hühnerblinddarm auffiel, dass das Pilokarpin nach Atropinisierung hemmend wirkt, so schien es mir von Interesse, diese neue Wirkung genau zu untersuchen. Als Versuchsmaterial benützte ich deshalb in erster Linie den ausgeschnittenen Hühnerblinddarm und versuchte, die Natur und das Wesen dieser neuen Wirkung klarzustellen. Anderseits bestrebte ich mich, diese Wirkung des Pilokarpins auch an anderem Versuchsmaterial nachzuweisen, und zwar am Dünndarm der Hühner und des Kaninchens und am Dickdarm des Kaninchens.

Die Versuchsmethode ist die gewöhnliche Suspensionsmethode. Als Nährflüssigkeit verwandte ich die Ringer-Lockesche Lösung, die stets auf $40^{\circ} \mathrm{C}$. gehalten wurde.

\section{Versuche am Hühnerblinddarm.}

\section{Vorbereitende Versuche.}

Die Bewegungen des Hühnerblinddarms sind im allgemeinen wenig lebhaft, aber wie die des Dünndarmes regelmässig.

Bevor wir auf die näheren Resultate mit Pilokarpin eingehen, empfiehlt es sich die Wirkungsweise verschiedener Nervenmuskelgifte auf den Hühnerblinddarm zu untersuchen, da dieses Organ-wie wir aus der Literatur ersehen-noch nie Objekt pharmakologischer Untersuchung gewesen ist, und deshalb von seiner Innervation noch nichts Sicheres bekannt ist, obwohl sich vermuten lässt, dass sie etwa derjenigen anderer Darmteile gleiche.

1) Adrenalin.

Es ist ganz bekannt, dass das Adrenalin infolge der Reizung des

1) Vergleiche, Fujita, M. ; Okayama Igakkai-Zasshi, Nr. 402, S. 507, 1923. 
Sympathikus auf den Tonus und die Bewegungen des Dünndarmes stark hemmend wirkt. Die Wirkung auf den Hühnerblinddarm ist fast dieselbe. Bei Einwirkung von 0,00001 g Adrenalin tritt stets eine deutliche Hemmung der Pendelbewegungen und des Tonus ein, welche aber bald wieder nachlässt. Diese Wirkung tritt um so deutlicher auf, je grösser die Dose ist. Diese hemmende Wirkung ist aber nicht dauernd. Selbst nach einer starken Hemmung durch über 0,0001 \% iges Adrenalin verschwindet sie nach einigen Minuten von selbst, sodass der herabgesetzte Tonus wieder fast bis auf sein normales Niveau zurückkehrt. Dabei konnte der Tonus durch 0,01-0,07 \%iges Pilokarpin noch gesteigert werden (Fig. 1).

2) Baryumchlorid.

Baryum in über 0,001 \%iger Lösung führt zu einer deutlichen Tonuszunahme, in 0,01-0,03 \%iger aber zu maximaler Kontraktion mit Erlöschen der Pendelbewegungen. Diese Kontraktion wird durch Atropin in keiner Weise gehemmt, wohl aber durch mittlere Dosen Adrenalin (wie $0,0001 \%$ ). Wenn die Kontraktion durch Baryum noch nicht vollkommen ist, so wirkt das Pilokarpin noch tonussteigernd, nicht aber bei maximaler Kontraktion durch das Baryum. Die Reaktion dieses Organs gegen Baryum lāsst sich also fast nicht von der anderer Dármteile unterschieden.

\section{3) Pilokarpin.}

0,0005-0,05 \%iges Pilokarpin bewirkt am Hühnerblinddarm immer eine starke Tonussteigerung, die nach höheren Dosen (wie 0,1 ) noch besträchtlicher auftritt (Fig. 2). In seltenen Fällen tritt aber nach Applikation von $0,1 \%$ igem Pilokarpin eine schnell vorübergehende Hemmung des Tonus auf (Fig. 3). Diese Hemmung ist aber in ihrem Verlaufe von der von Kondo $^{1)}$ und Fjitani $^{2)}$ nach 0,1 \% igem Pilokarpin am Darm, resp. am Magen des Frosches beobachteten verschieden.

Die deutliche Erregung, welche durch 0,005-0,05 \%iges Pilokarpin ausgelōst wird, geht fast völlig wieder zurück, wenn einige Minuten nach der Pilokarpinzufuhr 0,0005-0,005\% iges Atropin hinzugefügt wird (Fig.

1) Kondo, S.; 1. c.

2) Fujitani, Y.; 1. c. 
4). An einem durch Applikation einer grossen Pilokarpindose (wie 0,1) vergifteten Präparate konnte durch 0,001-0,005 \% iges Atropin noch ein sehr heftiger Tonusabfall verursacht werden, obgleich das Pilokarpin vorher keine deutliche Erregung gezeigt hatte (Fig. 3).

4) Atropin.

Die Wirkung von kleinen Atropindosen $(0,0005-0,004 \%)$ auf den Hühnerblinddarm ist eine sehr unbestimmte, d. h. es zeigt sich bald Hemmung, bald Erregung, bald keine Wirkung. Nach noch höheren Atropindosen $(0,005-0,05 \%)$ tritt in den meisten Fällen eine mehr oder weniger ausgeprāgte Tonuszunahme ein, nur ausnahmsweise eine Tonnusabnahme. Den antagonistischen Einfluss von kleinen Dosen Atropin gegenüber der Pilokarpinerregung habe ich schon bei der Wirkung des Pilokarpins erörtert.

Aus obigen Resultaten ersieht man, dass die Wirkungsweise dieser Gifte auf den Blinddarm der Hühner derjenigen auf den Dünndarm auch anderer Warmblüter sehr ähnlich ist. Er wird durch das sympathikuserregende Adrenalin gehemmt, durch das parasympathikuserregende Pilokarpin gefördert und durch das parasympathikuslāhmende Atropin gehemmt, wenn die Parasympathikusendigungen vorher durch Pilokarpin erregt worden sind.

Es kann also aus oben geschilderten Tatsachen mit höchster Wahrscheinlichkeit geschlosssen werden, dass die Innervation des Hühnerblinddarmes fast dieselbe ist wie die des Dünndarmes.

\section{Die Wirkung des Pilokarpins auf den atropinisierten Darm.}

Der durch 0,0005-0,005 \% iges Atropin vergiftete Hühnerblinddarm wird durch sonst erregende Dosen Pilokarpin deutlich gehemmt, gleichviel ob der Tonus des Darmes durch Atropin vorher gesteigert oder herabgesetzt worden ist. Diese hemmende Wirkung des Pilokarpins ist sogar auch an einem Darme, der durch eine noch höhere Dose Atropin (über $0,005 \%)$ in Erregung versetzt worden ist, ebenso deutlich zu beobachten. 
Der Grad der Hemmung des Pilokargins ist dabei nicht von den vorher angewandten Atropindosen abhängig, sondern nur von der Pilokarpindose. Er ist, wie in Fig. 5-8 gezeigt wird, um so grösser, je grösser die Dose ist. An dem Präparate, das 4 Minuten lang der Einwirkung von 0,001 $\%$ igem Atropin ausgesetzt war, verursacht 0,01\% iges Pilokarpin eine schwache Tonusabnahme, welche etwa 2 Minuten lang andauert (Fig. 5). Unter $0,01 \%$ verursacht das Pilokarpin aber dabei keine nennenswerte Hemmung mehr. Eine grōssere Dose Pilokarpin (wie 0,04) fühıt unter denselben Bedingungen eine noch beträchtlichere Tonusabnahme heıbei. Dabei bleiben sogar die Pendelbewegungen aus (Fig. 6). Wird die Pilokarpingabe bis auf $0,1 \%$ gesteigert, so dauert der herabgesetzte Tonus ziemlich lange Zeit an, während er durch Zusatz von 0,005-0,02\%iges Baryumchlorid leicht wieder gesteigert werden kann (Fig. 7).

Das Pilokarpin kann dagegen trotz der intensiven Erregung durch Baryum noch den Tonus bis zu einem gewissen Grade hemmen, wenn man den Darm vor dem Zusatz von Pilokarpin mit Atropin vergiftet hat (Fig. 8).

Bei wiederholter Applikation von Pilokarpin nach der Atropinisierung ist der Effekt je nach den gegebenen Pilokarpindosen verschieden. Wenn der Darm zuerst mit einer nicht zu hohen Dose Pilokarpin (z. B. 0,02$0,03 \%)$ vergiftet worden ist, so zeigt er nach der Erholung des Tonus bei dep zweiten Einwirkung dieses Giftes wieder eine erneute Hemmung, nicht aber, wenn. der Darm zuerst schon mit einer höheren Dose Pilokarpin (wie über $0,05 \%$ ) stark gehemmt. worden ist (Fig. 9). Es sei hier auch nebenbei bemerkt, dass sich die Stärke der hemmenden Wirkung des Pilokarpins wenige Minuten nach der Vorbehandlung mit Atropin kaum von der über eine halbe Stunde nach der Vorbehandlung unterscheidet.

Kurz, der ausgeschnittene Hühnerblinddarm wird nach der Vorbehandlung mit Atropin durch sonst erregende Dosen Pilokarpin stets gehemmt, und der Grad dieser Hemmung nimmt mit den Dosen zu.

Es fragt sich nun, worauf die hemmende Wirkung des Pilokarpins auf den atropinisierten Darm beruht. Diese Wirkung ist am Darm eine ganz neue Erșcheinung und lässt sich mit den bisherigen pharmakologischen Kenntnissen, dass dieses Gift die parasympatischen Nervenendigungen des 
Darms errege, nicht erklären. Es muss hier also noch einen anderen Angriffspunkt geben.

'Wenn ein Gift auf den ausgeschnittenen Darm hemmend wirkt, so kann das seine Ursache in der Reizung des hemmenden Sympathikus oder in der Lähmung der motorischen Ganglien oder des Muskels selbst haben. Dies zu entscheiden, ist aber eine schwere Aufgabe, denn wir haben bei unseren jetzigen Kenntnissen noch keine sichere direkte Methode, eine Erregung des hemmenden Sympathikus von einer unvollkommenen Lähmung des motorischen Apparates zu unterscheiden. Doch scheint mir aus folgenden Gründen die erstere Annahme, dass es sich hier um eine Reizung des Sympathikus handele, viel wahrscheinlicher.

Das Pilokarpin wirkt auf den intakten Blinddarm nur erregend und erst nach vor- oder nachheriger Atropinisierung hemmcnd. Es vermag auch nach solcher Vorbehandlung mit Atropin der durch Baryum verursachten Tonuszunahme entgegenzuwirken, wie das beim Adrenalin der Fall ist. Endlich ist auch der Darm, der durch Pilokarpin gehemmt ist, gegen Baryum noch gut emfindlich, und selbst nach einer so grossen Dose wie 0,1\% kann durch Baryum noch eine starke Kontraktion hervorgerufen werden.

Aus all diesen Tatsachen möchte ich schliessen, dass das Pilokarpin neben seiner eigentlichen Wirkung auf den Parasympathikus auch einewenn auch schwächere - reizende Wiı kung auf den hemmenden Sympathikus hat, dass aber diese Wirkung beim normalen Darm von jener ersten verdeckt wird und erst nach Lähmung der parasympáthischen Nervenendigungen durch Atropin zum Vorschein kommt.

Wie oben erwähnt, wird der zuerst durch grosse Dosen Pilokarpin gehemmte Darm nach der Erholung des Tonus durch eine zweite Dar-: reichung von Pilokarpin fast nicht mehr beeinträchtigt, während nach kleineren Dosen Pilokarpin der Darm durch eine weitere Dose eine erneute Hemmung erfährt. Das macht sehr wahrscheinlich, dass das Sympathikusende durch Pilokarpin zuerst gereizt und dann gelähmt wird, und dass deshalb, wenn man erst nur eine kleinere Dose Pilokarpin anwendet, der Sympathikus 'gegen eine folgende hemmende Dose Pilokarpin noch erregbar ist. Auch diese Erscheinungen scheinen die Annahme zu bestätigen, dass die genannte Wirkung des Pilokarpins sympathischer Natur ist. 
Wie oben erwähnt, zeigt das Adrenalin noch eine deutliche hemmende Wirkung auf den Darm, wo das Pilokarpin selbst bei wiederholter Applikation versagt (Fig. 9). Das beweist, dass der Angriffspunkt des Pilokarpins noch zentraler liegt als der des Adrenalins.

\section{Versuche am Darm anderer Tierarten.}

Wie bereits in meiner früheren Arbeit erwähnt worden ist, wird der atropinisierte Dünndarm der Hühner durch Pilokarpin fast in keiner Weise beeinflusst. Die hemmende Wirkung des Pilokarpins, wie man sie am Blinddarm sieht, kann hier nāmlich nicht beobachtet werden.

Auch der atropinisierte Dünndarm des Kaninchens wird, wie Kress gesehen hat, durch Pilokarpin im allgemeinen gar nicht beeinflusst, unter Umständen aber erfāhrt er eine mehr oder weniger starke Tonuszunahme. In seltenen Fällen konnte ich im Gegensatz zu den bisherigen Resultaten eine hemmende Wirkung des Pilokarpins wie beim Hühnerblinddarm konstatieren. Wie man in Fig. Io sehen kann, wurde der Darm, dessen Pendelbewegungen durch 0,02 \% iges Atropin etwas vergrössert worden waren, nach 6 Minuten durch Applikation von 0,05 \% igem Pilokarpin gehemmt, d. h. es folgten Tomusabnahme und Verkleinerung der Pendelbewegungen.

Ein anderes Beispiel bietet der Fall von Fig. 11, wo der durch Atropin gesteigerte Tonus durch nachherige Einwirkung von Pilokarpin etwas herabgesetzt wurde.

Auch am Dickdarm des Kaninchens ist die Wirkungsweise des Pilokarpins dieselbe wie am Dünndarm. Das Pilokarpin übt auf ihn nach der Atropinisierung gewöhnlich keinen Einfluss aus. Doch habe ich ausnahmsweise auch an diesem Organ eine mit dem Resultate am Hühnerblinddarm übereinstimmende Wirkung dieses Giftes beobachtet. Nach 8 Minuten langer Einwirkung von 0,002 \% igem Atropin wurde der Dickdarm durch 0,05 \% iges Pilokarpin gehemmt, wobei die Pendelbewegungen eine Weile ganz verschwanden und sich dann langsam erholten (Fig. 12).

Diese Tatsachen zeigen also, dass das Pilokarpin in seltenen Fällen auch am Dünndarm und Dickdarm des Kaninchens wie am Hühnerblind- 
darm nach Vorbehandlung mit Atropin eine hemmende Wirkung entfaltet.

Die Ursache des Zustandekommens dieser hemmenden Wirkung könnte man auf den jeweilig verschiedenen Zustand des betreffenden Organs zurückführen.

\section{Zusammenfassung.}

1. Der ausgeschnittene Blinddarm der Hühner zeigt gegen Adrenalin, Atropin, Pilokarpin und Baryum fast dieselbe Reaktion wie andere Teile des Darmes. Daraus wird mit voller Wahrscheinlichkeit geschlossen, dass die Innervation dieses Organs auch fast dieselbe ist wie die der anderen.

2. Das Pilokarpin ruft in ganz merkwürdiger Weise an dem mit Atropin vorbehandelten Blinddarm in sonst erregenden Dosen stets eine Hemmung des Tonus und der Bewegungen hervor.

3. Das Pilokarpin ist auch imstande, bei vorheriger Steigerung des Tonus durch Baryum denselben bis zu einem gewissen Grade zu hemmen. Dagegen zeigt der durch grössere Dosen Pilokarpin stark gehemmte Darm immer noch eine fast normale Empfindlichkeit gegen Baryum.

4. Obige Tatsachen machen es mir sehr wahrscheinlich, dass die Hemmung des Pilokarpins auf eine Erregung der hemmenden Sympathikusendigungen im Darm zurückzuführen ist.

5. Nach einer starken Hernmung durch eine grosse Dose Pilokarpin kann eine nochmalige Zufuhr von Pilokarpin keine Hemmung mehr verursachen. Daraus wird gefolgert, dass die Sympathikusendigungen durch Pilokarpin zwar erst erregt, dann aber gelähmt werden.

6. Da bei diesem Versagen des Pilokarpins das Adrenalin noch eine hemmende Wirkung entfalten kann, so muss der Angriffspunkt des Pilokarpins noch zentraler liegen als der des Adrenalins.

7. Auch am Dünn- und Dickdarm des Kaninchens, nicht aber am Hühnerdünndarm, wurde in einzelnen Fällen ein ähnlicher hemmender Einfluss des Pilokarpins nachgewiesen, der vermutlich auch auf denselben Mechanismus zurückgeführt werden kann. 
Fig. 1.

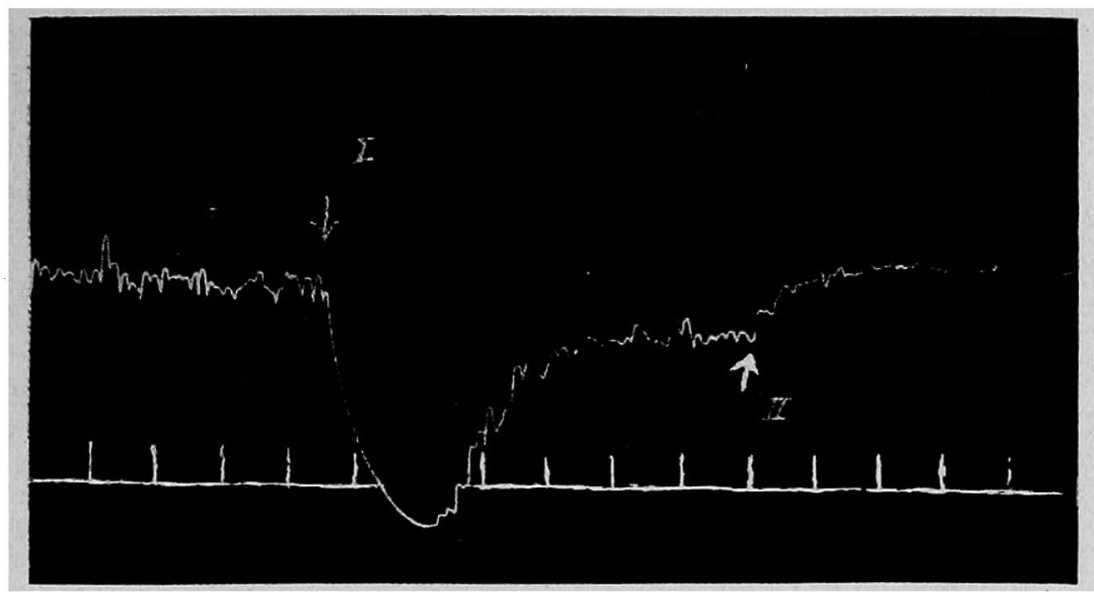

Hühnerblinddarm.

I $=0,0001 \%$ Adrenalin. hydrochlor.

II $=0,02 \%$ Pilocarpin. hydrochlor.

Zeitmarken je 1 Minute.

Fig. 2.

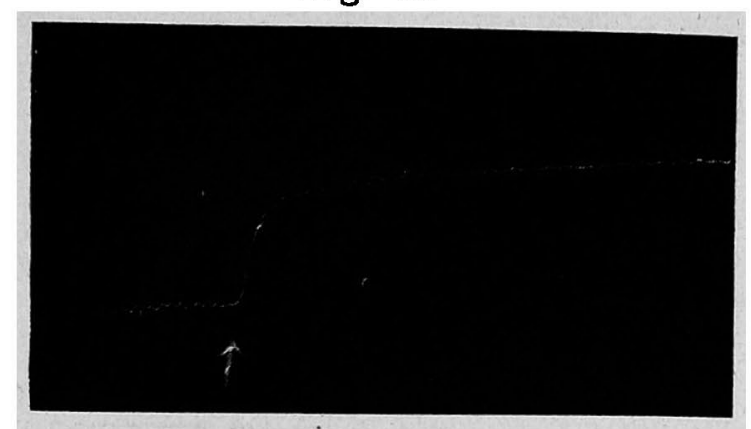

Hühnerblinddarm.

$\uparrow=0,1 \%$ Pilocarpin. hydrochlor.

Fig. 3.

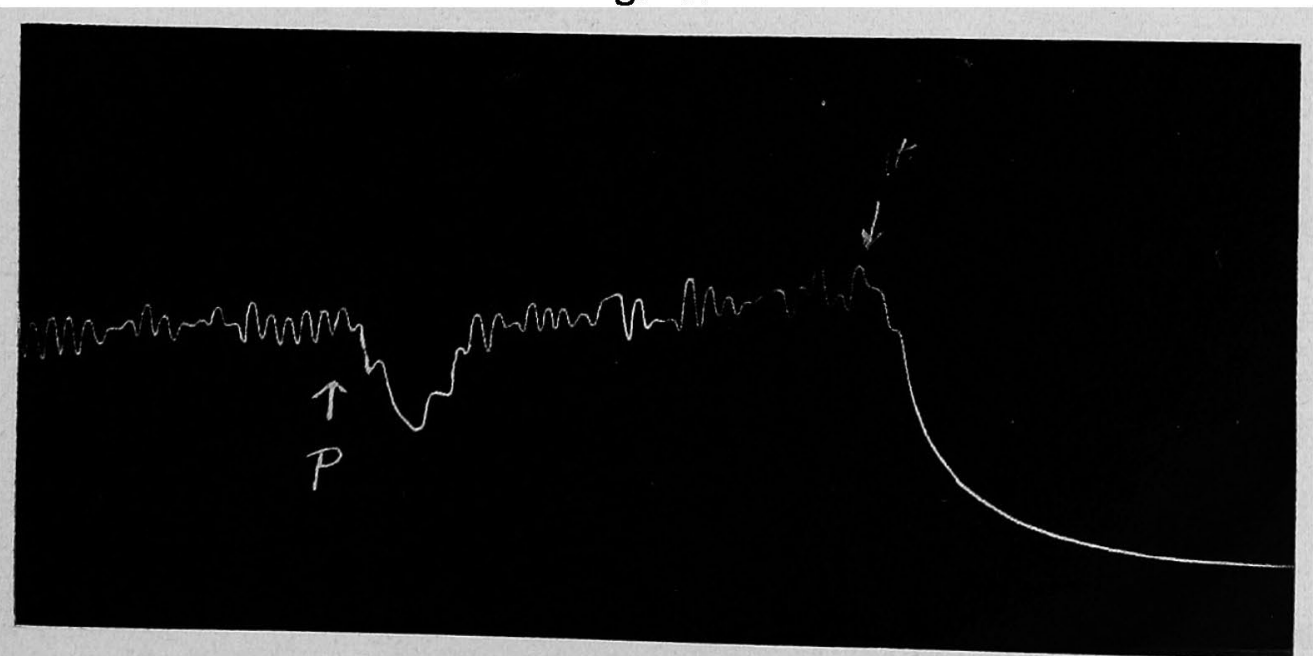

Hühnerblinddarm.

$P=0,1 \%$ Pilocarpin. hydrochlor. $\quad$ At $=0,005 \%$ Atropin. sulf. 
Fig. 4.

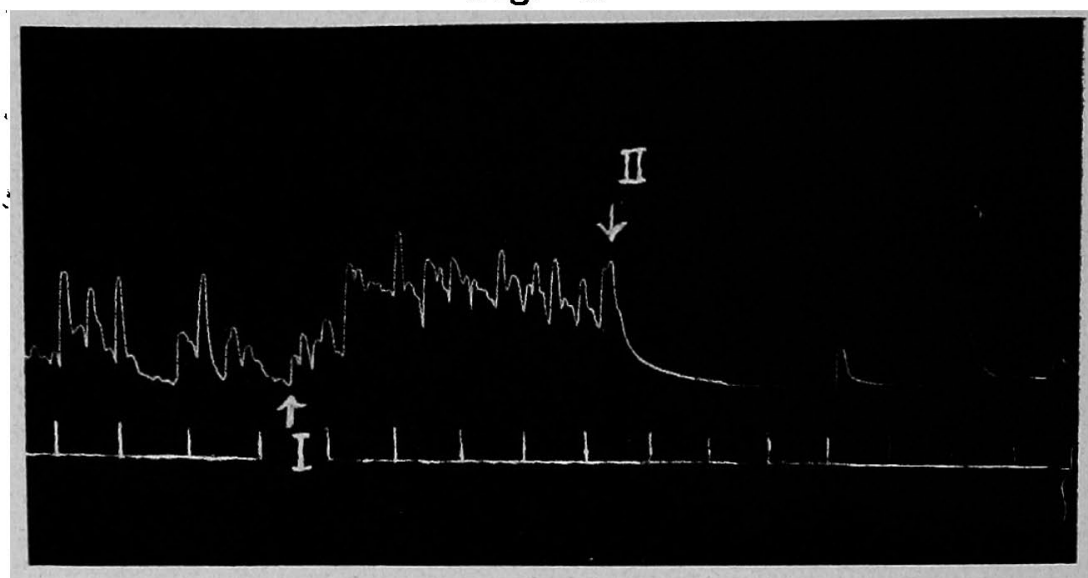

Hühnerblinddarm.

I $=0,005 \%$ Pilocarpin. hydrochlor. $\quad$ II $=0,001 \%$ Atropin. sulf. Zeitmarken je 1 Minute.

Fig. 5.

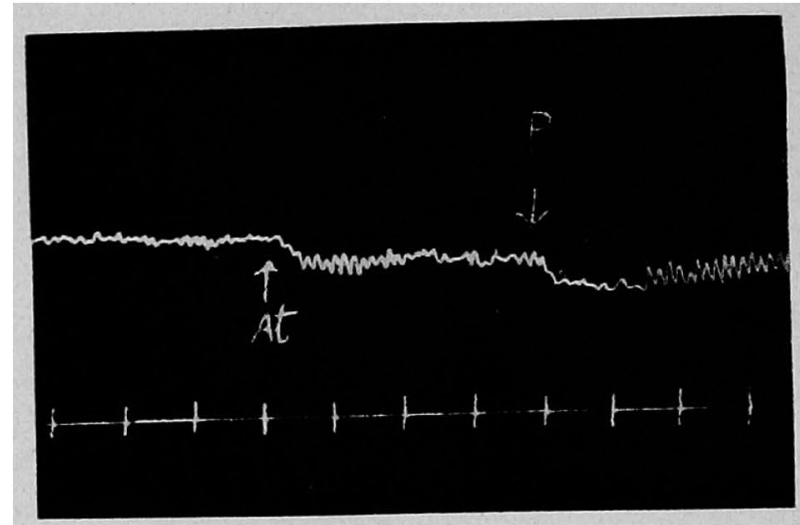

Hübnerblinddarm.

At $=0,001 \%$ Atropin. sulf. $\quad \mathbf{P}=0,01 \%$ Pilocarpin. hydrochlor.

Zeitmarken je 1 Minute.

Fig. 6.

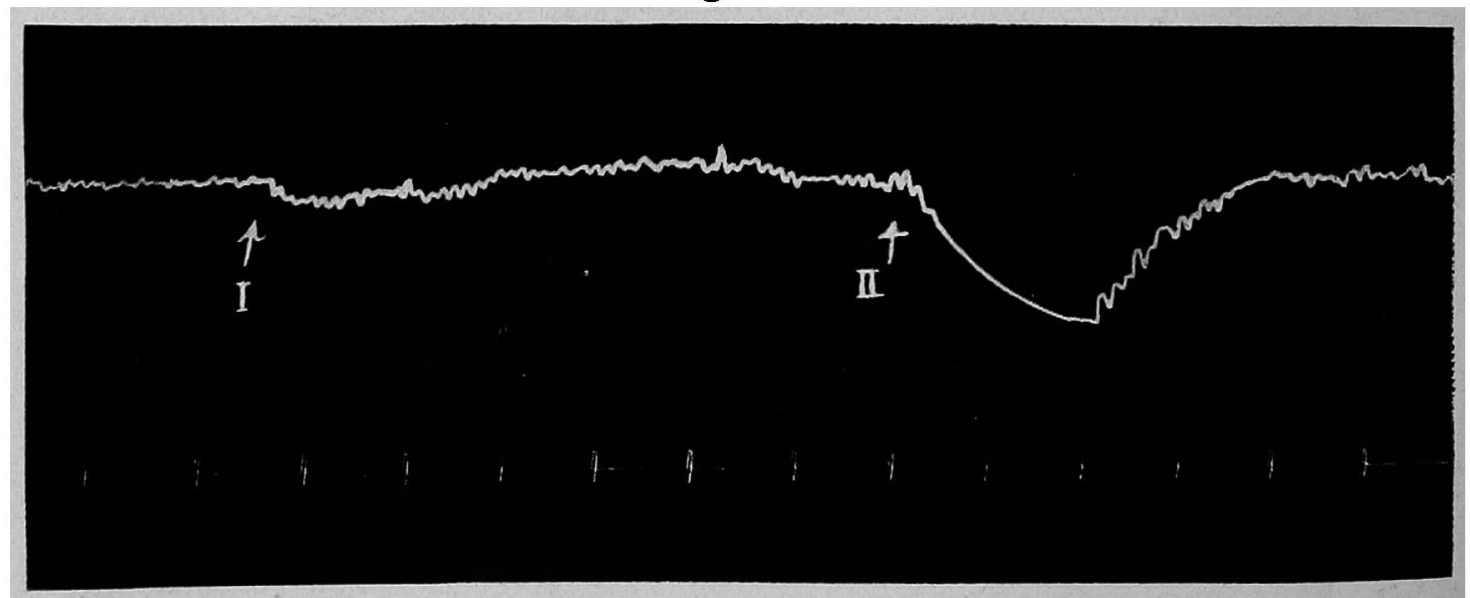

Hühnerblinddarm.

I $=0,004 \%$ Atropin. sulf. $\quad$ II $=0,04 \%$ Pilocarpin. hydrochlor. $\quad$ Zeitmarken je 1 Minute. 
Fig. 7.

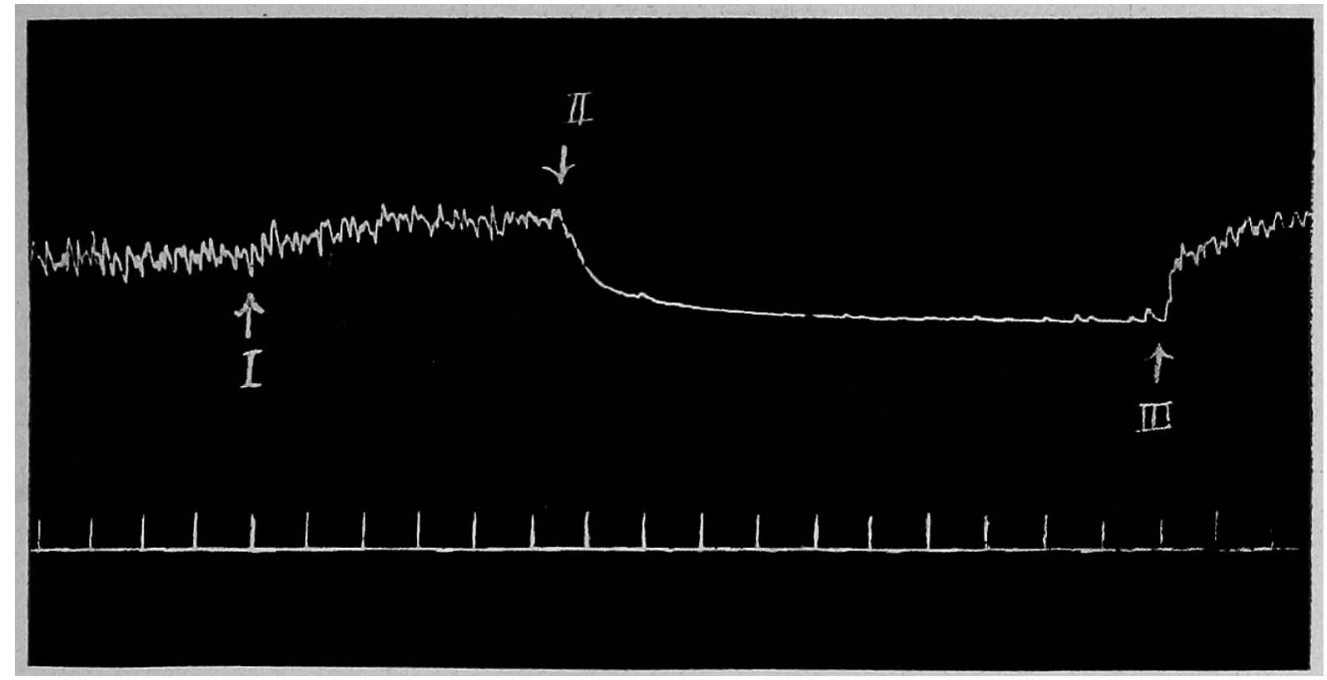

Hühnerblinddarm.

$$
\begin{aligned}
\mathrm{I} & =0,015 \% \text { Atropin. sulf. } \\
\mathrm{II} & =0,1 \% \text { Pilocarpin. hydrochlor. } \\
\mathrm{III} & =0,01 \% \text { Barium. chlorat. } \\
& \text { Zeitmarken je } 1 \text { Minute. }
\end{aligned}
$$

Fig. 8.

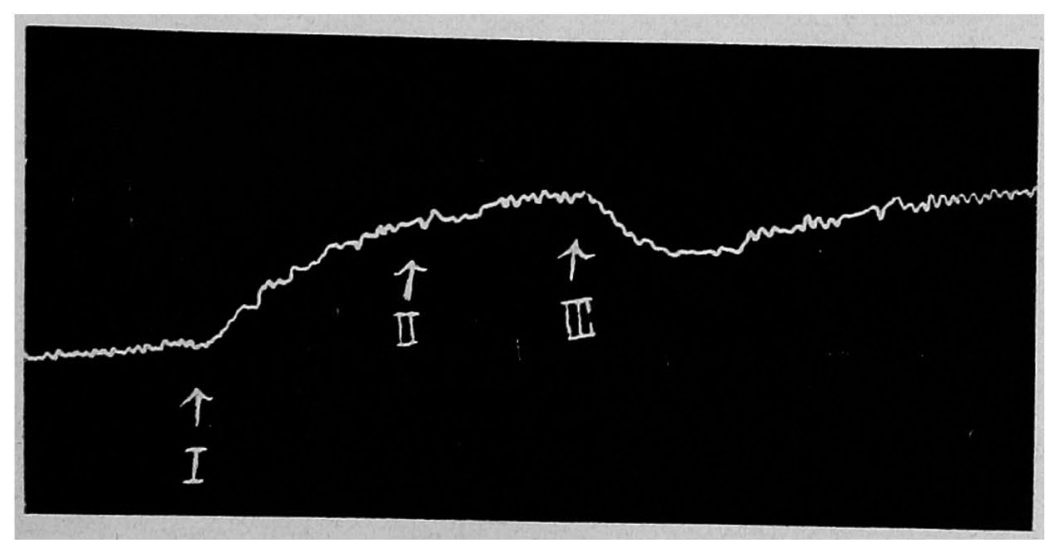

Hühnerblinddarm.

$\mathrm{I}=0,01 \%$ Barium. chlorat.

II $=0,01 \%$ Atropin. sulf.

III $=0,05 \%$ Pilocarpin. hydrochlor. 


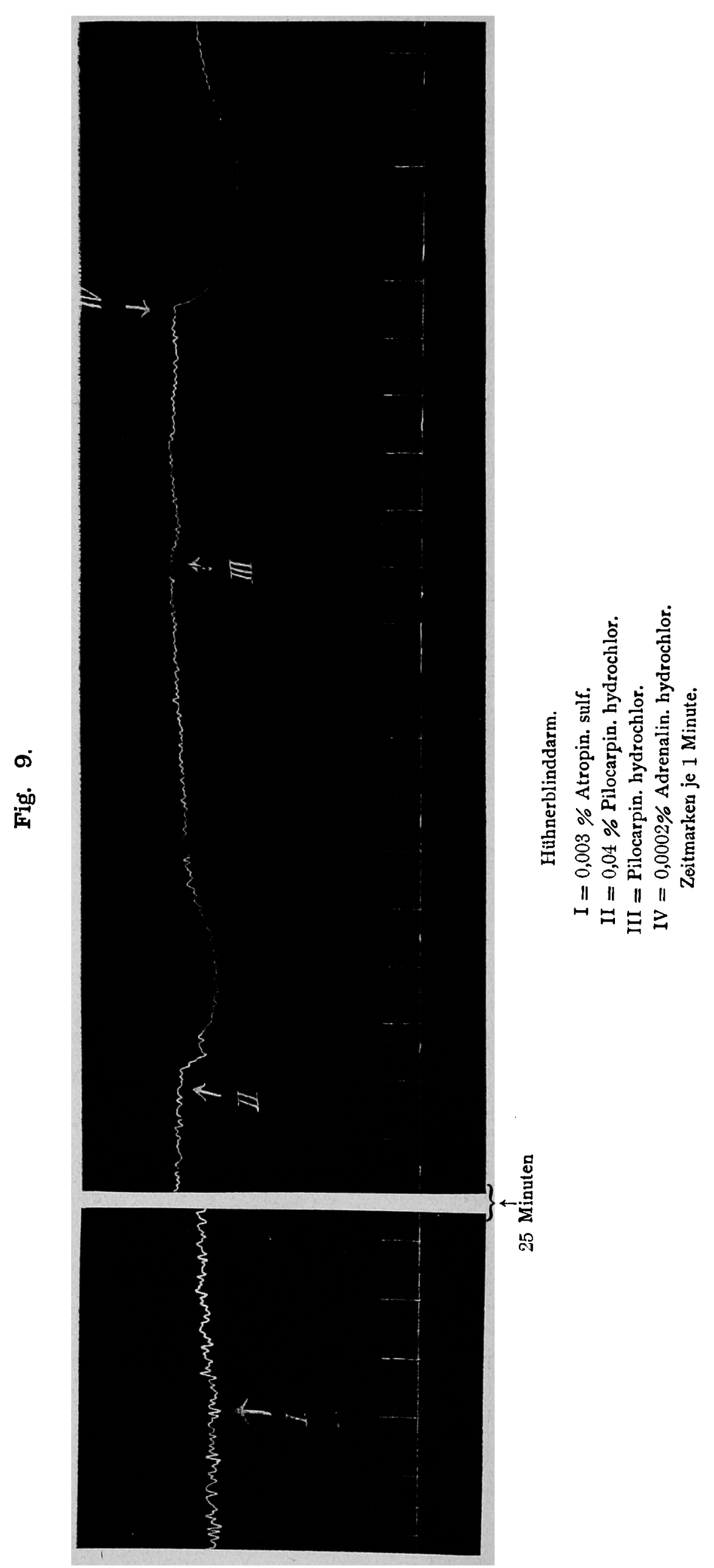



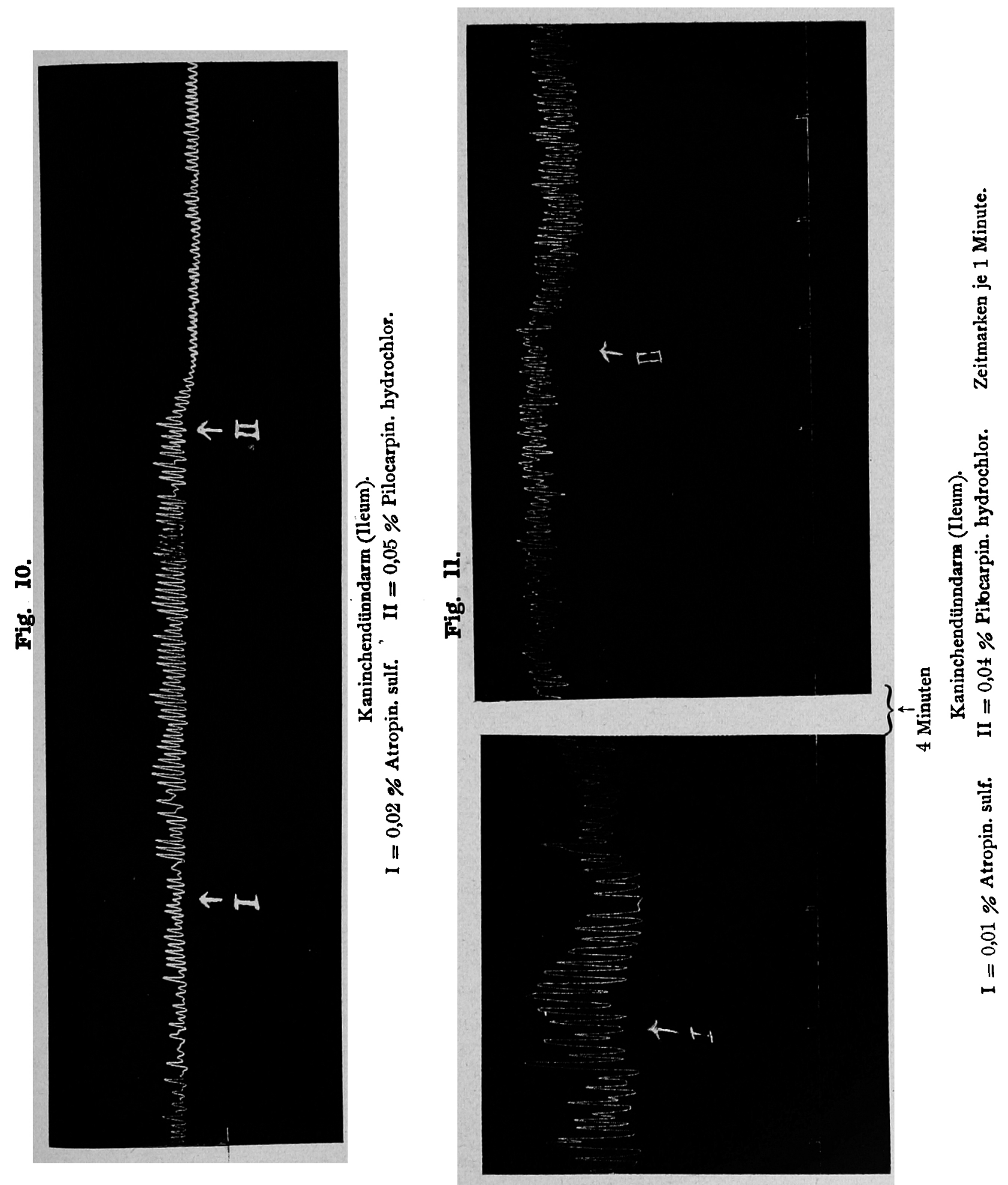


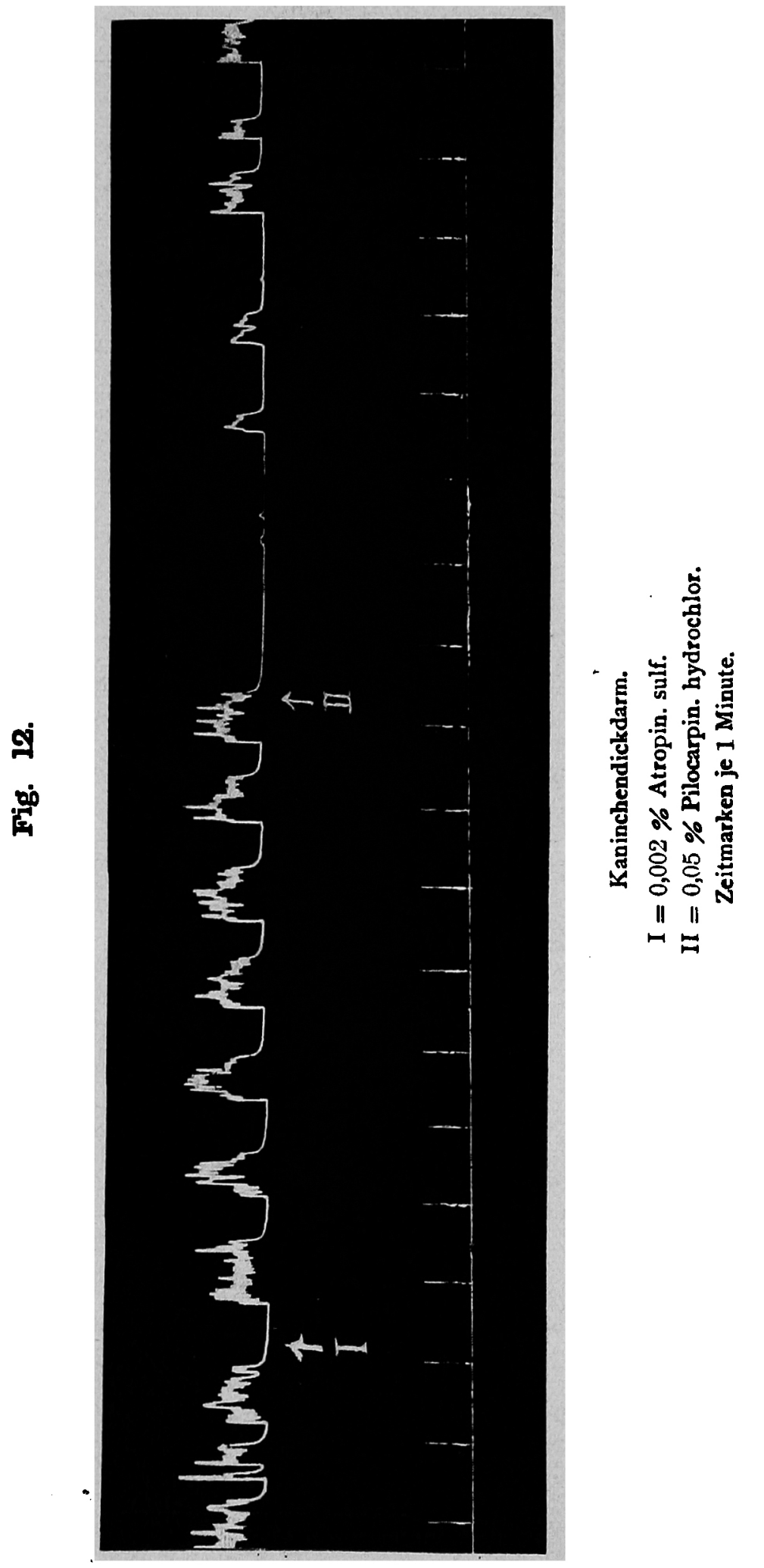

